\title{
ESTRÉS LABORAL Y CUIDADO DE ENFERMERIA, CENTRO QUIRURGICO DEL HOSPITAL AUGUSTO HERNANDEZ MENDOZA, ICA- 2016
}

Labor stress and nursing care, surgical center of Hospital Augusto Hernández Mendoza, Ica- 2016 Amanda María García Aquije1,2,a,b

${ }^{1}$ Facultad de Enfermería de la Universidad Nacional San Luis Gonzaga de Ica ${ }^{2}$ Hospital Augusto Hernández Mendoza - Essalud, Ica.

aLicenciada en Enfermería, 'bMagíster en Salud Pública

\section{RESUMEN}

El presente estudio, tuvo por objetivo analizar la relación entre el estrés laboral y el cuidado de enfermería en el Centro Quirúrgico del Hospital Augusto Hernández Mendoza, de Ica, durante el año 2016. Material y métodos: Estudio descriptivo, no experimental, correlacional con diseño transversal, muestra 17 enfermeros y 75 pacientes del centro quirúrgico. Como instrumentos se utilizaron el cuestionario The Nursing Stress Scale para determinar el nivel de estrés en los enfermeros (34 preguntas), y el cuestionario Care $Q$ para determinar la percepción del paciente sobre el cuidado de enfermería recibido (41 preguntas). Las variables se relacionaron mediante la prueba de Chi Cuadrado y correlación bivariada de Pearson. Resultados: El 47,1\% de los enfermeros tiene estrés alto, $23,5 \%$ estrés medio y $29,4 \%$ estrés bajo. Los más altos índices de estrés de nivel alto, se estarían produciendo por falta de apoyo $(35,3 \%)$, problemas con otros miembros de enfermería (35,3\%), y preparación insuficiente $(23,5 \%)$. En cuanto a la percepción del cuidado: el $52,9 \%$ de los pacientes percibe un buen cuidado por parte del enfermero, y un $47,1 \%$ un mal cuidado. Los más alto índice de buen cuidado de enfermería, se observan en las dimensiones: Monitorea y hace seguimiento $(88,2 \%)$, accesibilidad $(64,7 \%)$, y explica y facilita (52,9\%); y en menor proporción el buen cuidado estaría sucediendo en las dimensiones: Forma en que conforta $(41,2 \%)$ y mantiene relación de confianza $(47,1 \%)$. Chi cuadrada señala relación estadística significativa entre las variables de estudio $\left(x^{2} 7,0, p, 000\right)$. Conclusiones: Existe relación estadística significativa entre estrés laboral y el cuidado de enfermería.

Palabras clave: Estrés laboral, cuidado de enfermería, pacientes, centro quirúrgico.

\section{SUMMARY}

The present study aimed to objective analyze the relationship between work stress and nursing care at the Augusto Hernández Mendoza Hospital Surgical Center in Ica during the year 2016. Material and methods: Descriptive, non-experimental, correlational study with design Transversal, shows 17 nurses and 75 patients of the surgical center. The Nursing Stress Scale questionnaire was used to determine the level of stress in nurses (34 questions) and the Care Q questionnaire to determine the patient's perception of the nursing care received (41 questions). Variables were correlated using the chi-square test and Pearson's bivariate correlation. Results: $47.1 \%$ of nurses have high stress, $23.5 \%$ mean stress and $29.4 \%$ low stress. The highest levels of high-level stress would be due to lack of support (35.3\%), problems with other nurses (35.3\%), and insufficient preparation $(23.5 \%)$. Regarding the perception of care: $52.9 \%$ of patients perceive good care by the nurse, and $47.1 \%$ poor care. The highest index of good nursing care is observed in the dimensions: It monitors and does follow up (88.2\%), accessibility (64.7\%), and explains and facilitates (52.9\%); And to a lesser extent good care would be happening in the dimensions: How it comforts (41.2\%) and maintains a relationship of trust $(47.1 \%)$. Chi square indicates statistically significant relationship between the study variables $(x 27.0, p, 000)$. Conclusions: There is a significant statistical relationship between work stress and nursing care.

Key words: Occupational stress, nursing care, patients, surgical center. 


\section{INTRODUCCIÒN}

La investigación titulada: "Estrés Laboral y el Cuidado de Enfermería, Centro Quirúrgico del Hospital Augusto Hernández Mendoza, de Ica, 2016; tuvo como propósito analizar la relación entre las variables estrés laboral y cuidado de enfermería, durante el 01 de enero al 01 de marzo del año 2016.

Las enfermeras, en el día a día nos enfrentamos a diversos factores y presiones propias de la profesión, desarrollamos diversas actividades siendo la más importante el cuidado de los pacientes, labor cada vez más estresante debido a su complejidad, a la mayor demanda de salud, tanto a nivel local, regional y nacional, lo que exige al personal de enfermería de mucha preparación, y vocación para el servicio.

Al respecto De La Cuesta (2007) (1), señala al cuidado como "una relación y un proceso cuyo objetivo va más allá de la enfermedad", entonces cuidar puede considerarse como un quehacer multidisciplinario que requiere habilidades, destrezas y afrontamiento de diversas situaciones estresantes dentro de una institución de salud.

En el ámbito de la salud, se ha probado en diversos estudios que el personal de Enfermería, es susceptible a desarrollar estrés, por encontrarse laborando dentro de un ambiente emocionalmente tóxico, permanentemente sometido a situaciones de interacción con pacientes y familiares, que según Basset (2011) (2), "propician el desarrollo de diversos niveles de estrés, tanto de carácter organizacional como propios de la profesión". En el área quirúrgica, las enfermeras están propensas a diversas situaciones estresantes y de presión propia de las intervenciones quirúrgicas y cuidados postoperatorios al paciente, lo cual influye considerablemente en el cuidado al paciente, causando algún déficit en la atención y cuidado de enfermería hacia los pacientes.
Por las consecuencias que pueda representar la presencia del estrés laboral en el personal de enfermería, es necesario identificarlo a tiempo, y tomar medidas para mitigar, dotándoles por ejemplo de guías de autoayuda. Según Mingote (2014) (3), ello implicaría adiestrar en el reconocimiento del estrés y ofrecerles herramientas para el control del mismo, lo que les permitirá desarrollar con satisfacción su tarea profesional y mejorar el cuidado de enfermería.

En los últimos años, se ha percibido como problemática el deficiente desempeño profesional según (Cogollo M. 2010) (4), "influenciado por diversos problemas psicoemocionales que repercuten considerablemente en el estilo de vida de los trabajadores", evidenciado también en diversas instituciones que prestan servicios de salud en nuestro país.

En la praxis, el personal de salud, se enfrenta día a día a diversos agentes estresores que repercuten en su desempeño profesional, en desmedro de la buena atención y cuidado que prestan a los pacientes durante el proceso de la enfermedad, recuperación, o postoperatorio.

Romero M. (2008) (5) y Novoa M. (2005) (6), han recopilado información de entidades internacionales como la Organización Mundial de la Salud (OMS), el Consejo Internacional de Enfermería (CIE) y la Organización Internacional del Trabajo (OIT), y aseguran que estas entidades señalan la importancia del trabajo de enfermería en el campo de la salud, al tiempo que han identificado condiciones de trabajo inadecuadas que la afectan, tales como los sistemas de salud en Latinoamérica mal implementados, el incremento de la carga laboral, la pérdida de seguridad laboral, la falta de suministros para la prestación de servicios, los bajos salarios, la falta de recurso humano de enfermería y el incremento de procesos judiciales, que constituyen factores que generan sobrecarga 
laboral, cansancio y agotamiento físicomental en este grupo laboral.

Según Salazar A. (2008) (7), el personal de enfermería se desenvuelve en múltiples contextos; uno de ellos es el centro quirúrgico, definido como el conjunto de ambientes físicos que proporcionan al equipo quirúrgico las facilidades necesarias para efectuar procedimientos quirúrgicos en forma eficaz, eficiente y en condiciones de máxima seguridad. Salazar señala que "el estado del paciente, su vulnerabilidad, el riesgo que implica el procedimiento anestésico y quirúrgico, sumados a la simultaneidad de tareas que enfrenta el equipo de enfermería, hacen que su trabajo sea estresante; por tanto, el servicio de cirugía requiere de un equipo de enfermería que pueda trabajar bajo estrés".

En el área quirúrgica, se encuentran numerosos factores que provocan estrés relacionados con los aspectos ambientales, ergonómicos y psicosociales, Viñez J. (1989) (8), manifiesta que en especial "las enfermeras instrumentistas o quirúrgicas, realizan buena parte de su jornada laboral en posición de pie, con pocas posibilidades de movimiento, con la atención centrada en las exigencias de la intervención quirúrgica y las indicaciones del cirujano".

El problema del estrés ha sido estudiado durante muchos años, considerándose un problema social, físico y psicológico; actualmente sigue vigente la necesidad de seguir estudiándolo. Diversas investigaciones señalan a los profesionales de enfermería, como a uno de los grupos más afectados por el estrés en su trabajo diario.

Moncayo M. (2009) (9), señala "en el contexto del centro quirúrgico, la misión de la enfermera quirúrgica no se limita a la instrumentación quirúrgica sino que tiene una tarea más compleja como es la de asistir, controlar, supervisar y evaluar todo el proceso transquirúrgico preservando la integridad y seguridad del paciente que ingresa al centro quirúrgico"

Cuando se manifiesta el estrés en los profesionales de enfermería que laboran en los Centros Quirúrgicos, en ocasiones afloran reacciones de diversa naturaleza en el desempeño de sus labores, que afectan entre otros, el cuidado de enfermería al paciente que ha sido intervenido quirúrgicamente, durante los años de servicio lo hemos percibido, $y$ es objeto de preocupación.

Por tanto, analizar la percepción del paciente sobre el cuidado de enfermería, determinar la autopercepción del estrés por parte del personal de enfermería, y analizar la relación entre estas variables, constituye el objetivo de la presente investigación: Analizar la relación entre el estrés laboral y el cuidado de enfermería en el Centro Quirúrgico del Hospital Augusto Hernández Mendoza, de Ica, durante el año 2016.

Los resultados de la investigación orientaran la toma de decisiones y permitirán plantear mecanismos de afrontamiento y solución a esta problemática que consideramos de importancia, como afirma Morales C. (2007) (10), que le permitan al personal de enfermería, enfrentar con cierta facilidad las situaciones de conflicto que enfrenta durante su quehacer profesional de modo que no repercuta en la calidad de servicio que brinda al usuario.

\section{MATERIAL Y MÉTODOS.}

La investigación es de tipo no experimental, nivel descriptivo correlacional, y diseño transversal

Muestra: a) Enfermeras: La muestra fue intencional, compuesta por 17 enfermeras (81\%), que laboraron en el centro quirúrgico del Hospital Augusto Hernández Mendoza de Ica (HAHM),que desearon participar proporcionando información en forma voluntaria. De las 21 enfermeras (100\%), 2 se encontraban de vacaciones, una con 
licencia y una no respondió el cuestionario. b) Pacientes: La muestra lo constituyeron 75 pacientes operados con cirugía mayor de baja complejidad, en el centro quirúrgico del HAHM, del 01 de febrero al 01 de marzo del 2016, quienes aceptaron participar voluntariamente proporcionando información para la investigación.

Para el recojo de información se aplicó el método inductivo, como técnicas fueron utilizadas la encuesta al personal de enfermería, y la entrevista a los pacientes; además de la observación el análisis y la síntesis. Como instrumentos se utilizaron: Para el estrés laboral el "The Nursing Stress Scale", cuestionario elaborado por Gray-Toft y Anderson, y para el cuidado de enfermería el Care Q (Caring Assessment Instrument), creado por Patricia Larson y Ferketich (1981).

La validez de los instrumentos NSS y Care- $Q$ se determinaron mediante la participación de 5 expertos investigadores en ciencias de la salud con el grado de doctor, de quienes se obtuvo altos índices de 0,96 y 0,98 respectivamente. El análisis de la confiabilidad de los instrumentos NSS y Care$Q$ se llevó a cabo mediante alfa de cronbach, habiéndose obtenido valores de 0,93 y 0,95 respectivamente.

Los índices mencionados representan alto grado de validez y confiabilidad en los instrumentos mencionados. Los datos fueron almacenados electrónicamente, procesados en Excel, exportados a SPSS v21,0, se presentan tablas, gráficos, para su mejor análisis, las variables se relacionaron mediante Chi Cuadrada y $r$ de Pearson para $95 \%$ de confianza

\section{RESULTADOS.}

En la tabla 1 se observa que casi la mitad $(47,1 \%)$ de las enfermeras en estudio presentan un alto estrés, $29,4 \%$ un bajo estrés y un $23,5 \%$ un mediano estrés.
Tabla 1. Estrés del personal de enfermería del Centro Quirúrgico del Hospital Augusto Hernández Mendoza

\begin{tabular}{lcc}
\multirow{2}{*}{ ESTRÉS } & \multicolumn{2}{c}{ FRECUENCIA } \\
\cline { 2 - 3 } & $N^{\circ}$ & $\%$ \\
\hline Alto & 8 & 47,1 \\
Medio & 4 & 23,5 \\
Bajo & 5 & 29,4 \\
\hline \multicolumn{1}{c}{ TOTAL } & 17 & 100,0 \\
\hline
\end{tabular}

La tabla 2 muestra las dimensiones del estrés, identificando que las enfermeras del presente estudio presentan un alto estrés: $35,3 \%$ por falta de apoyo y problemas con otros miembros de enfermería. Así mismo se tiene que más de la mitad: $52,9 \%$ de dichas enfermeras presentan un mediano estrés.

Tabla 2. Dimensiones del estrés del personal de enfermería del Centro Quirúrgico del Hospital Augusto Hernández Mendoza

\begin{tabular}{|c|c|c|c|c|c|c|c|c|}
\hline \multirow{2}{*}{$\begin{array}{c}\text { DIMENSIONES DEL } \\
\text { ESTRÉS }\end{array}$} & \multicolumn{2}{|c|}{ ALTO } & \multicolumn{2}{|c|}{ MEDIO } & \multicolumn{2}{|c|}{ BAJO } & \multicolumn{2}{|c|}{ TOTAL } \\
\hline & $\mathrm{N}^{\circ}$ & $\%$ & $\mathrm{~N}^{\circ}$ & $\%$ & $\mathrm{~N}^{\circ}$ & $\%$ & $\mathrm{~N}^{\circ}$ & $\%$ \\
\hline Carga de trabajo & 3 & 17,6 & 7 & 41,2 & 7 & 41,2 & 17 & 100,0 \\
\hline $\begin{array}{l}\text { Ambiente psicológico: } \\
\text { Muerte y sufrimiento }\end{array}$ & 3 & 17,6 & 4 & 23,5 & 10 & 58,8 & 17 & 100,0 \\
\hline Preparación insuficiente & 4 & 23,5 & 4 & 23,5 & 9 & 52,9 & 17 & 100,0 \\
\hline Falta de apoyo & 6 & 35,3 & 7 & 41,2 & 4 & 23,5 & 17 & 100,0 \\
\hline $\begin{array}{l}\text { Incertidumbre en el } \\
\text { tratamiento }\end{array}$ & 2 & 11,8 & 9 & 52,9 & 6 & 35,3 & 17 & 100,0 \\
\hline $\begin{array}{l}\text { Problemas con los } \\
\text { médicos }\end{array}$ & 3 & 17,6 & 7 & 41,2 & 7 & 41,2 & 17 & 100,0 \\
\hline $\begin{array}{l}\text { Problemas con otros } \\
\text { miembros de } \\
\text { enfermería }\end{array}$ & 6 & 35,3 & 3 & 17,6 & 8 & 47,1 & 17 & 100,0 \\
\hline
\end{tabular}

En la tabla 3 se observa el cuidado de enfermería percibido por los pacientes del Centro Quirúrgico, determinado según sus dimensiones.

Tabla 3. Cuidado de enfermería según sus dimensiones

\begin{tabular}{lcc}
\multicolumn{1}{c}{$\begin{array}{c}\text { Dimensiones del } \\
\text { cuidado }\end{array}$} & Bueno & Malo \\
\cline { 2 - 3 } & $\%$ & $\%$ \\
\hline Accesible & $64,7 \%$ & $35,3 \%$ \\
\hline Explica - Facilita & $52,9 \%$ & $47,1 \%$ \\
\hline Conforta & $41,2 \%$ & $58,8 \%$ \\
\hline Anticipa & $58,8 \%$ & $41,2 \%$ \\
\hline Relación - Confianza & $47,1 \%$ & $52,9 \%$ \\
\hline Monitorea & $88,2 \%$ & $11,8 \%$ \\
\hline
\end{tabular}


Con una probabilidad de error del 5\% la prueba estadística ji cuadrado determina que existe relación entre el estrés del personal de enfermería y el cuidado que brinda a los pacientes. En enfermería, el cuidado se considera como la esencia de la disciplina que implica no solamente al receptor, sino también a la enfermera como transmisora de él. En la tabla 4 se observa que $29,4 \%$ de este personal en estudio, con un bajo estrés, realiza un buen cuidado; así mismo se tiene que el $35,3 \%$ con un alto nivel de estrés realiza un mal cuidado.

Tabla 4. Estrés y cuidado del personal de enfermería del Centro Quirúrgico del Hospital Augusto Hernández Mendoza

\begin{tabular}{lcccccc}
\hline & \multicolumn{4}{c}{ CUIDADO DE } & \multirow{2}{*}{ ENFERMERÍA } & \multirow{2}{*}{ TOTAL } \\
\cline { 2 - 6 } ESTRÉS & \multicolumn{3}{c}{ BUENO } & \multicolumn{2}{c}{ MALO } & \\
\cline { 2 - 6 } & $\mathrm{N}^{\circ}$ & $\%$ & $\mathrm{~N}^{\circ}$ & $\%$ & $\mathrm{~N}^{\circ}$ & $\%$ \\
\hline Alto & 2 & 11,8 & 6 & 35,3 & 8 & 47,1 \\
Medio & 2 & 11,8 & 2 & 11,8 & 4 & 23,5 \\
Bajo & 5 & 29,4 & 0 & 0,0 & 5 & 29,4 \\
\hline TOTAL & 9 & 52,9 & 8 & 47,1 & 17 & 100,0 \\
\hline \multicolumn{1}{c}{ g.I.(2) } & \multicolumn{5}{c}{$\mathrm{X}^{2}{ }_{\mathrm{c}}=7.0>\mathrm{X}^{2}{ }_{\mathrm{t}}=5,991$}
\end{tabular}

\section{DISCUSIÓN}

Estrés Laboral. Gonzáles B, (2013) (11), señala que "tener estrés es estar sometido a una gran presión, sentirse frustrado, aburrido, encontrarse en situaciones en las que no es fácil el control de las mismas, tener problemas conyugales, etc", afirmación que se complementa con la de Lazarus y Folkman (1984) (12), "el estrés es una relación entre la persona y el ambiente, en la que el sujeto percibe en qué medida las demandas ambientales constituyen un peligro para su bienestar, si exceden o igualan sus recursos para enfrentarse a ellas", en este enfoque los estudios de Cortaza L. y Francisco M. (2014)(13), concluyen que los enfermeros se perciben a sí mismos como personas estresadas, con sobrecarga de trabajo y sensibles al sufrimiento de los usuarios, $y$ encontraron que $100 \%$ de enfermeros presentaban algún nivel de estrés, coincidiendo con Barrios $H$. y
Carvajal D. (2010) (14), y con los hallazgos del presente estudio, que señalan en forma global, $47,1 \%$ de enfermeros con estrés de nivel alto, $29,4 \%$ estrés de nivel bajo, y $23,5 \%$ estrés de nivel medio; de acuerdo al análisis realizado, los niveles de estrés alto se estarían produciendo por problemas con otros miembros de enfermería (35,3\%), falta de apoyo $(35,3 \%)$ o preparación insuficiente $(23,5 \%)$, también se pueden explicar debido a la edad de las enfermeras 46,8 años $(41,1 \%$ son mayores de 51 años de edad), tiempo de servicio como enfermera: $35,3 \%$ tienen entre 25 a 40 años de servicio, 23,5\% trabajan entre 23 a 36 años en el centro quirúrgico, $17,6 \%$ de enfermeras tienen a personas discapacitadas a su cargo; y $47,1 \%$ son casadas y $88,2 \%$ tienen que cumplir múltiples funciones en el hogar; así mismo coinciden con Cortaza L. y Francisco M. (2014) (13) quienes encontraron que el aspecto más afectado en el personal de enfermería fue el físico, siendo la carga de trabajo el factor que genera más estrés, seguido de la muerte y el sufrimiento, así como la incertidumbre en el tratamiento; además con Barrios H. y Carvajal D. (2010)(14), que relacionaron la presencia de estrés laboral debido a la sobrecarga de trabajo, la inseguridad y el miedo a cometer errores.

Por otra parte Coris R. (2010) (15), hallo $29 \%$ de enfermeras con nivel alto de estrés, quienes presentan síntomas de agotamiento emocional, mencionan que trabajar directamente con personas les produce estrés, muestran preocupación por el trabajo que les endurezca emocionalmente, y se sienten cansados al final de la jornada de trabajo; situaciones que repercuten en la calidad de atención de enfermería. Gutiérrez S. y Revatta R. (2014) (16), en Pisco,Perú, en centros quirúrgicos de dos Hospitales de EsSalud ha encontrado estrés laboral alto en $28,6 \%$ de las enfermeras, (cifra menor al presente estudio 47,1\%), que estaría provocando niveles graves de agotamiento 
emocional, despersonalización y realización personal.

En cuanto a las dimensiones del estrés se ha encontrado altos niveles en: Carga de trabajo $(17,6 \%)$, muerte y sufrimiento $(17,6 \%)$, preparación insuficiente $(23.5 \%)$, falta de apoyo (35,3\%), incertidumbre en el tratamiento $(11,8 \%)$, problemas con los médicos $(17,6 \%)$, y problemas con otros miembros de enfermería (35,3\%), índices que son necesarios tomar en cuenta, a fin de asegurar la salud de las enfermeras, y la buena atención hacia los pacientes, mientras exista altos niveles de estrés laboral menor será el nivel de cuidado de enfermería.

Cuidado de enfermería. De la cuesta (2007) (1), señala que "cuidar es una actividad humana que se define como una relación $y$ un proceso cuyo objetivo va más allá de la enfermedad"; por otra parte. Watson J. (1979) (17), afirma que "el cuidado se manifiesta en la práctica interpersonal, que tiene como finalidad promover la salud y el crecimiento de la persona". En enfermería, el cuidado se considera como la esencia de la disciplina que implica no solamente al receptor, sino también a la enfermera como transmisora de él. Con estos enfoques se han llevado a cabo diversas investigaciones sobre el cuidado de enfermería, inclusive la presente investigación. Los hallazgos de la investigación señalan, en promedio $52,9 \%$ de pacientes que perciben buen cuidado de enfermería, y 47,1\% mal cuidado; el estudio de Campos L; Chico J, y Cerna L. (2015) (18), en Trujillo, indica una cifra mayor de buen cuidado (70\%), Rojas Martínez, y Barajas Lizarazo (2012) (19), en España, hallaron niveles medianos de calidad de cuidado de enfermería; resultados que difieren debido a los contextos donde se realizaron las investigaciones, a los instrumentos de recojo de información utilizados (Ficha de observación-Care Q), y a la población de estudios. En cuanto a las dimensiones del Instrumento aplicado, el más alto índice de buen cuidado, se observa en la dimensión Monitorea y hace seguimiento $(88,2 \%)$ accesibilidad $(64,7 \%)$, y explica y facilita $(52,9 \%)$; en menor proporción en las dimensiones: Forma en que conforta $(41,2 \%)$ y relación de confianza $(47,1 \%)$.

\section{Características sociodemográficas de los} enfermeros. Se encontró proporción mayoritaria de mujeres $94,1 \%$, edad promedio $\bar{X} 46,76$ años (DS 11,56), predominan los grupos etarios de 42 a 50 años (23,5\%) y 58 a 64 años (23,5\%). En cuanto al estado civil, el $47,1 \%$ son casados, $41,2 \%$ solteros; $41,2 \%$ tienen de 9 a 16 años de servicio, 23,5\% registran de 1 a 8 años de servicios como enfermeros y $23,5 \%$ de 25 a 32 años de servicio, $29,4 \%$ tienen 2 o 3 hijos respectivamente, $17,6 \%$ tienen a personas discapacitadas a su cargo, $88,2 \%$ realizan tareas en el hogar antes o después de laborar como enfermeros, 29,4\% trabajan entre 2 a 8 años y 16 a 22 años en el centro quirúrgico. En cuanto a la situación laboral $64,7 \%$ son contratados y $35,3 \%$ nombrados.

Características sociodemográficas de los pacientes. Se encontró predominio del género femenino $(57,3 \%)$, y del grupo etario de 28 a 37 años $(30,7 \%)$. La edad promedio fue $\bar{X} 41,18$ años (DS 11,83), 37,3\% son casados, $36 \%$ convivientes, $100 \%$ de los pacientes poseen seguro de salud, $38,6 \%$ tienen de 3 a 5 hijos, 98,7\% están activos laboralmente. En cuanto a las patologías que presentaron al momento de intervención quirúrgica 22,6\% fueron cesáreas, 10,4\% fracturas, $\quad 9,3 \% \quad$ colecistectomía laparoscópica, 6,7\% apendicectomía, 5,2\% laparotomías, entre otros, Asimismo 58,6\% son de Ica, $10,6 \%$ de sus provincias, y $30,8 \%$ proceden de sus distritos urbanos y rurales.

\section{CONCLUSIONES}

1. Existe relación estadística significativa entre estrés laboral y el cuidado de enfermería. Mientras exista altos niveles 
de estrés laboral menor será el nivel de cuidado de enfermería.

2. En forma global, $47,1 \%$ de enfermeros observaron estrés laboral de nivel alto, $29,4 \%$ estrés de nivel bajo y $23,5 \%$ presentaron estrés de nivel medio.

3. Según dimensiones del estrés, se ha encontrado altos niveles en: Falta de apoyo $(35,3 \%)$, problemas con otros miembros de enfermería (35,3\%), y preparación insuficiente $(23,5 \%) ; y$ en menor proporción se encontró altos niveles en: carga de trabajo $(17,6 \%)$, ambiente psicológico (17,6\%), problemas con los médicos $(17,6 \%)$ e incertidumbre de trabajo $(11,8 \%)$.

4. En forma global, $52,9 \%$ de los pacientes señalaron que el cuidado de enfermería fue bueno, y $47,1 \%$ afirmaron que fue malo el cuidado de enfermería.

5. Según dimensiones del cuidado de enfermería, se ha encontrado niveles de buen cuidado en: Accesibilidad (64,7\%), explica y facilita (52,9\%), conforta $(41,2 \%)$, se anticipa $(58,8 \%)$, relación de confianza $(47,1 \%)$, y monitorea y hace seguimiento (88,2\%). Existe relación significativa entre el estrés y las dimensiones del cuidado, tales como: Se anticipa $\left(x^{2} 7,70\right)$ y relación de confianza $\left(x^{2} 9,50\right)$.

\section{RECOMENDACIONES}

1. Se sugiere sensibilizar a las enfermeras y a la institución de salud, sobre la naturaleza y ocurrencia del estrés laboral: Delimitar funciones, rotar personal, fomentar actividades físicas y deportivas, estímulos, renovar e incrementar personal de enfermería y capacitación permanente.

2. Mejorar la calidad del cuidado de enfermería, tomando como referencia la percepción de los pacientes, como un sistema de mejora continua.

3. Mejorar el clima laboral en el servicio del Centro Quirúrgico, entre el personal de salud, integrándolos, socializándolos, propiciando buenas relaciones interpersonales.

4. Mejorar la gestión de Recursos Humanos, analizar e identificar factores que al relacionarse con otras variables socio laborales y sociodemográficas provocan estrés en las enfermeras, que repercuten en su misión de cuidar adecuadamente a los pacientes

5. Continuar la investigación en todos los servicios del hospital involucrando a todo el personal de salud, a pacientes de todos los servicios, y ampliando la muestra.

\section{Correspondencia:}

Mg. Amanda García Aquije

Correo electrónico:

agarciaaquije@hotmail.com

Celular: 956019220

\section{REFERENCIAS BIBLIOGRÁFICAS}

1. De la Cuesta C. El cuidado del otro: Desafíos y posibilidades. Medellín, Colombia. Rev. Invest. educ. enferm . 2007; 25(1):106-112.

2. Basset I, Estévez R, et al. Estrés laboral y personal en los recursos humanos de Enfermería de una Unidad de Psiquiatría en México, D.F. Rev. Enf Neurol .2011; 10 (1): 27-31.

3. Mingote J; Perez S. Estrés en la enfermería: El cuidado del cuidador. Madrid, España: Ed. Díaz de Santo; 2014.

4. Cogollo M, Bustamante G. Condiciones laborales en enfermeras de Cartagena Colombia, 2010.Rev. Avances en Enfermería.2010; 23 (1) :3138

5. Romero M, Mesa L, Galindo S. Calidad de vida de las enfermeras y sus consecuencias para el cuidado .Bogotá, Colombia .Rev. Avances en Enfermería; 2008; 26 (2): 59-70 
6. Novoa M, Nieto C, Forero C, Caicedo C, Palma M, Montealegre $\mathrm{M}$, et al. Relación entre Perfil Psicológico, calidad de vida y estrés asistencial en personal de enfermería. 2005. Rev Univ Psychol; (4) 1:63-75

7. Salazar A. Cirugía: Un contexto diferente de cuidado. Bogotá, Colombia .Rev. Avances en Enfermería.2011; 29(1):55-66.

8. Viñez J. Exposición a riesgos. Sociedad Española de Higiene y Medicina Preventiva Hospitalaria. España; 1989.

9. Moncayo $\mathbf{M}$, Oña $\mathbf{N}$. Gestión de enfermería en el proceso de atención quirúrgica intraoperatoria del Hospital Militar de las Fuerzas Armadas. Quito, Ecuador; 2009.

10. Morales $\mathbf{C}$. Nivel de estrés laboral de las enfermeras que laboran en las áreas críticas del Instituto Nacional de Salud del Niño. Noviembre-Diciembre. 2006 Tesis Pregrado. Lima, Perú. Facultad de Medicina. Escuela Académico Profesional de Enfermería, Universidad Nacional de San Marcos; 2007.

11. Gonzáles B, Parada J, Guzmán W. Prevalencia del estrés laboral y su correlación con la conducta asertiva en los empleados de la farmacia Brasil, ubicada en Av. Cacahuatique 40 Bis, colonia Chaparrastique de la ciudad de San Miguel, durante el año 2013. Tesis Licenciatura. Brasil. Universidad de EI Salvador; 2013.

12. Lazarus, R.S. y Folkman, S. Estrés y procesos cognitivos. Barcelona, España; 1986.

13. Cortaza L, Francisco M. Estrés laboral en enfermeros de un hospital de Veracruz.Mexico.Rev.Aladefe.2014;4(1).
14. Barrios H, Carbajal D. Estrés en el personal de enfermería del área de Emergencia Adulto, en el Hospital Ruiz y Páez, Ciudad de Bolívar - Edo Bolívar. Tesis Pregrado. Universidad del Oriente. Cumaná, Venezuela; 2010.

15. Coris R. Nivel de Estrés Laboral de las Enfermeras de Centro Quirúrgico del Hospital Essalud de Huancavelica. Tesis. Huancavelica, Perú; 2010.

16. Gutiérrez S, Revatta R. Estrés laboral en profesionales de enfermería que laboran en los Centros Quirúrgicos de los Hospitales San Juan de Dios y Antonio Skrabonja Antoncich de Pisco 2013. Tesis Segunda Especialidad. Ica, Perú.Facultad de Enfermería, Universidad Nacional San Luis Gonzaga de Ica.; 2014.

17. Watson J. Nursing: The Philosophy and Science of caring. Boston: Little Brown and Company; 1979, p. 320

18. Campos L,Chico J, Cerna L. Calidad del cuidado de enfermería en la seguridad integral del paciente quirúrgico del Hospital Base Víctor Lazarte Echegaray, Trujillo 2014. Trujillo, Perú. Rev. SCIÉNDO Ciencia para el Desarrollo.2015; 17(1).

19. Rojas M, y Barajas L. Percepción de la calidad del cuidado de enfermería en el servicio de urgencias, de la Unidad Básica Puente Barco Leones de La Pamplona en España. Rev. Ciencia y Cuidado. 2012 ; 9(1) :13-23

Recibido: 20/11/17

Aprobado para Publicación: 06/03718 